\title{
Dynamics of the Upper Oceanic Layers in Terms of Surface Quasigeostrophy Theory
}

\author{
G. LAPEYRE AND P. KLEIN \\ Laboratoire de Physique des Océans, IFREMER, Plouzané, France
}

(Manuscript received 26 October 2004, in final form 11 July 2005)

\begin{abstract}
In this study, the relation between the interior and the surface dynamics for nonlinear baroclinically unstable flows is examined using the concepts of potential vorticity. First, it is demonstrated that baroclinic unstable flows present the property that the potential vorticity mesoscale and submesoscale anomalies in the ocean interior are strongly correlated to the surface density anomalies. Then, using the invertibility of potential vorticity, the dynamics are decomposed in terms of a solution forced by the three-dimensional (3D) potential vorticity and a solution forced by the surface boundary condition in density. It is found that, in the upper oceanic layers, the balanced flow induced only by potential vorticity is strongly anticorrelated with that induced only by the surface density with a dominance of the latter. The major consequence is that the $3 \mathrm{D}$ balanced motions can be determined from only the surface density and the characteristics of the basin-scale stratification by solving an elliptic equation. These properties allow for the possibility to reconstruct the 3D balanced velocity field of the upper layers from just the knowledge of the surface density by using a simpler model, that is, an "effective" surface quasigeostrophic model. All these results are validated through the examination of a primitive equation simulation reproducing the dynamics of the Antarctic Circumpolar Current.
\end{abstract}

\section{Introduction}

Many studies in the last 10 years have been devoted to the understanding of the dynamical properties of the potential temperature anomalies on the tropopause (see Hakim et al. 2002, and references therein). Motivation is that much of the dynamics in the upper levels of the troposphere is influenced by the movements of the tropopause, and the latter are intimately correlated with the temperature anomalies there (Juckes 1994). Most of these studies rely on the well-known invertibility principle of potential vorticity (PV) detailed in the seminal paper of Hoskins et al. (1985). This principle, widely used in atmospheric studies but much less often in oceanography, allows one to diagnose the 3D dynamics of a balanced flow [such as that studied by Gent and McWilliams (1983), among others] from the knowledge of PV at different levels and temperature at the boundaries. Such an approach for the tropopause dynamics is all the more relevant for mesoscale and submesoscale structures at these levels as these structures

Corresponding author address: G. Lapeyre, LMD/IPSL, Ecole Normale Supérieure, 75005 Paris, France.

E-mail: glapeyre@lmd.ens.fr are mostly influenced by PV at the upper levels of the troposphere near the tropopause layer. One outcome is that the 3D dynamics of the upper troposphere can be well described from only the potential temperature anomalies on the tropopause (Juckes 1994) using surface quasigeostrophy (SQG) theory (Held et al. 1995).

In this study we follow a similar approach within an oceanographic context and examine the role of the surface boundary condition in the dynamics of the upper oceanic layers. The backbone of our approach is the invertibility principle mentioned before. The full PV inversion problem involving the 3D distribution of PV and the surface density is posed and discussed in the next section. We decompose the flow in two categories associated with different PV sources: a first contribution comes from the inversion of the interior PV field while a second contribution arises from the PV inversion of the density field at the surface. Then in section 3 we highlight an important property of baroclinic flow in that PV mesoscale and submesoscale anomalies are correlated with surface density anomalies in space. As a consequence, the interior PV and surface density contributions to the $3 \mathrm{D}$ dynamics are strongly dependent on each other. Last, in section 4 we propose a method to reconstruct the mesoscale and submesoscale dynam- 
ics of the upper layers from just the knowledge of the surface density.

\section{Quasigeostrophic PV inversion}

The PV inversion problem relies on two requirements. First, one needs to know the spatial distribution of the 3D PV field with appropriate boundary conditions. Second, a system of balanced equations has to be chosen since this choice determines the inversion technique to be used (McIntyre and Norton 2000; Vallis 1996). Here we assume that the balanced flow is in quasigeostrophic (QG) equilibrium with a small Rossby number and a Burger number of order 1. This assumption means that the PV inversion is used to get mesoscale and submesoscale motions, that is, oceanic motions with time scales of 10-30 days and spatial scales of $10-200 \mathrm{~km}$.

\section{a. Decomposition into PV sources}

The knowledge of PV and surface density allows one to deduce the $3 \mathrm{D}$ circulation through the principle of invertibility (Bishop and Thorpe 1994; Hoskins et al. 1985). In QG theory, PV is related to the streamfunction by an elliptic operator,

$$
\nabla^{2} \psi+\frac{\partial}{\partial z}\left(\frac{f_{0}^{2}}{N^{2}} \frac{\partial \psi}{\partial z}\right)=Q,
$$

where $\psi$ is the streamfunction, $f_{0}$ is the Coriolis frequency, and $N$ is the Brunt-Väisälä frequency; $\boldsymbol{\nabla}$ is the horizontal gradient operator, and $z$ is the vertical coordinate. Here $Q$ is defined as the anomaly from the largescale planetary PV. To invert the elliptic operator, one also needs appropriate boundary conditions, in particular at the ocean surface:

$$
-\left.\frac{\rho_{0} f_{0}}{g} \frac{\partial \psi}{\partial z}\right|_{z=0}=\left.\rho\right|_{z=0} .
$$

Here $\rho$ is the density anomaly. If we introduce a buoyancy variable $b=-g \rho / \rho_{0}$, we have

$$
\left.f_{0} \frac{\partial \psi}{\partial z}\right|_{z=0}=\left.b\right|_{z=0} .
$$

For the lower boundary conditions, we use $\partial_{z} \psi=0$ at the ocean bottom. We assume that through the PV inversion process the dynamics of the upper layers is mostly captured by the PV distribution in these layers; that is, the low-level PV anomalies have little effect on the upper-layer dynamics. For the lateral boundary condition, we consider as a simplification that the domain is doubly periodic.

In QG theory, PV and surface buoyancy are both conserved by the advection along the geostrophic flow $\mathbf{u}=(u, v)=\left(-\partial_{y} \psi, \partial_{x} \psi\right)$,

$$
\begin{aligned}
\frac{D Q}{D t} & =0 \text { and } \\
\frac{D_{s} b_{s}}{D t} & =0,
\end{aligned}
$$

where $D / D t=\partial_{t}()+\mathbf{u} \cdot \boldsymbol{\nabla}()$ and the subscript $s$ applies to surface quantities (i.e., at $z=0$ ).

The inversion consists in solving (1) and (3) to find the streamfunction $\psi$ and the buoyancy that satisfies the QG hydrostatic equation

$$
b=f_{0} \frac{\partial \psi}{\partial z} .
$$

Mathematically, one can split this inversion into two different problems [this idea goes back to Bretherton (1966)]. The first one (noted "int" for interior) is forced by nonzero PV in the interior and involves zero surface buoyancy:

$$
\nabla^{2} \psi_{\text {int }}+\frac{\partial}{\partial z}\left(\frac{f_{0}^{2}}{N^{2}} \frac{\partial \psi_{\text {int }}}{\partial z}\right)=Q
$$

with

$$
\left.f_{0} \frac{\partial \psi_{\text {int }}}{\partial z}\right|_{z=0}=0 .
$$

The second one (noted "sur" for surface) involves zero $\mathrm{PV}$ in the interior and is forced by nonzero surface buoyancy:

$$
\nabla^{2} \psi_{\text {sur }}+\frac{\partial}{\partial z}\left(\frac{f_{0}^{2}}{N^{2}} \frac{\partial \psi_{\text {sur }}}{\partial z}\right)=0
$$

with

$$
\left.f_{0} \frac{\partial \psi_{\text {sur }}}{\partial z}\right|_{z=0}=\left.b\right|_{z=0} .
$$

Coming back to the full problem, the total streamfunction field is given by the sum of the two contributions,

$$
\psi=\psi_{\text {int }}+\psi_{\text {sur }},
$$

by the principle of superposition. One should take care that the total streamfunction is needed to advect both PV and surface buoyancy in (4a)-(4b).

\section{b. Dynamical interpretation}

The following dynamical interpretation of the two problems, (4a) and (6a)-(6b) on one hand and (4b) and (7a)-(7b) on the other hand, is proposed. The first one resembles the classical QG model (e.g., such as the Phil- 
lips model) governed by the dynamics of interior PV layers: when a large-scale PV forcing is included in (4a), it is the change in sign on the vertical of the horizontal gradient of PV that is responsible for the baroclinic instability and the mesoscale structures that emerge. The second problem is associated with frontogenesis (although ageostrophic mechanisms such as outcropping are absent in the QG approximation) because the advection of buoyancy (4b) (even when a surface buoyancy forcing is included) leads to the stirring of buoyancy contours and to the development of horizontal fronts at the surface. When taking (7a)-(7b) and (4b) as a single system (with $\psi=\psi_{\text {sur }}$ ), we obtain the surface quasigeostrophic (SQG) model (Blumen 1978; Hakim et al. 2002; Held et al. 1995), which describes the evolution of edge waves that decay exponentially from the surface. The SQG model is a reduction of the Eady model to the surface and therefore does not allow baroclinic instability.

The combination of the SQG model with the interior PV model can be thought of in terms of the Charney model [which involves nonzero PV interior and a nonzero surface buoyancy (Pedlosky 1987)]. This suggests that the two problems can strongly interact. Actually, different studies (Davis 1993; Green 1987; Hakim et al. 1996; Holopainen and Kaurola 1991) have questioned the independence between a flow generated by the surface buoyancy and a flow generated by the interior PV. As buoyancy is involved in the calculation of PV, the two solutions may not be independent from each other. Holopainen and Kaurola (1991) find at atmospheric large scales that the geopotentials induced by the surface buoyancy and interior PV tend to cancel each other. Hakim et al. (1996) obtain a similar finding in studying a cyclogenesis event. On the other hand, the relationship between the two solutions might be associated with a dynamical feature. Indeed two studies have shown that cancellation is not always the general case: Davis and Emanuel (1991) in a cyclogenesis event do not observe a cancellation. Also Robinson (1989) found that, in the Charney model of linear baroclinic instability, the cancellation of PV fluxes due to the interior PV with those due to the surface buoyancy depends on the wavelength considered. In our study, to understand the dynamical relationship between the surface and interior solutions, we thus need to analyze the properties of these solutions within the framework of baroclinic instability since this mechanism is the major source of mesoscale structures in the ocean.

\section{Properties of the surface/interior decomposition}

We first demonstrate that under appropriate assumptions common for the ocean, surface buoyancy and PV in the interior are correlated spatially. Such a correlation has been observed in the atmosphere (Holopainen and Kaurola 1991), and one of the reasons invoked by the authors was that the buoyancy field is used in the definition of PV so that the surface buoyancy and the PV near the surface are able to be correlated in the presence of a forcing in buoyancy. Here we show that the reason is more dynamical in nature. Then we deduce the impact of this correlation on the relation between the surface and interior solutions.

\section{a. Relation between surface buoyancy and interior $P V$}

We start from the observation that eddy PV and buoyancy anomalies are forced by the baroclinic instability of a large-scale flow. As the specific large-scale forcings of the two quantities are similar, the anomalies tend to correlate to each other because the two tracers are stirred by the eddies in the same manner. This scenario closely resembles the one described by Klein et al. (1998), which explains the thermohaline anomalies compensated in density by the stirring effects of the eddies. To understand this correlation, we first split surface buoyancy and PV into the zonal mean (i.e., large scale) and a deviation (noted respectively by overbarred quantities and primes). The equations for the anomalies are

$$
\begin{aligned}
& \frac{D Q^{\prime}(x, y)}{D t}=-v \frac{\partial \bar{Q}}{\partial y} \text { and } \\
& \frac{D_{s} b_{s}^{\prime}(x, y)}{D t}=-v_{s} \frac{\partial \bar{b}_{s}}{\partial y} .
\end{aligned}
$$

To derive these equations, we assumed the zonally averaged PV and surface buoyancy to be slowly varying in time. The large-scale meridional PV gradient can be developed into three different terms:

$$
\frac{\partial \bar{Q}}{\partial y}=\beta-\frac{\partial^{2} \bar{u}}{\partial y^{2}}+\frac{1}{f_{0}} \frac{\partial}{\partial z}\left(\frac{f_{0}^{2}}{N^{2}} \frac{\partial \bar{b}}{\partial y}\right) .
$$

The first two terms correspond to the mean meridional gradients of planetary and relative vorticities. The last term is the mean meridional gradient of the vortex stretching associated with the nonconstant stratification on the vertical and the curvature of the mean shear. Let us assume that the spatial distribution of $\bar{b}$ is separable,

$$
\bar{b}(y, z) \approx \bar{b}_{s}(y) F(z)
$$

with $F(z)=1$ at $z=0$. This can be motivated by the fact that, in the ocean, often the first baroclinic mode dominates. The first two terms in (10) are usually small so that the mean PV gradient can be written as propor- 
tional to the mean buoyancy gradient at the ocean surface; that is,

$$
\frac{\partial \bar{Q}}{\partial y} \approx \frac{1}{f_{0}} \alpha(z) \frac{\partial \bar{b}_{s}}{\partial y}
$$

with

$$
\alpha(z)=\frac{\partial}{\partial z}\left[\frac{f_{0}^{2} F(z)}{N(z)^{2}}\right]
$$

related to the vertical structure of $\bar{b}$. Potential vorticity is advected only by the horizontal velocity field at all depths in QG theory, while surface buoyancy is also advected by the horizontal velocity field evaluated at the surface. We assume that the horizontal velocity field varies little on the vertical (at least for the first few hundreds of meters) since most of the associated kinetic energy is usually captured by the barotropic and first baroclinic modes (Hua and Haidvogel 1986; Smith and Vallis 2001). One can then assume that, at a first order, potential vorticity at a depth $z$ and surface buoyancy are advected by the same velocity field. Then, by (12a) and (9a)-(9b), an equation for conservation along the geostrophic flow is obtained:

$$
\frac{D \mathcal{T}}{D t}=0
$$

with

$$
\mathcal{T}=Q^{\prime}-\frac{1}{f_{0}} \alpha b_{s}^{\prime} .
$$

There is no source to create $\mathcal{T}$, contrary to $Q^{\prime}$ and $b_{s}^{\prime}$, which are forced by mean gradients. So, by the straining action of eddies it will cascade to small scales where it will be dissipated after some time. Stating $\mathcal{T} \approx 0$, we thus obtain a direct relation between interior PV anomalies and surface buoyancy anomalies,

$$
Q^{\prime}(x, y, z) \approx \frac{1}{f_{0}} \alpha(z) b_{s}^{\prime}(x, y) .
$$

This important result indicates that, in baroclinic flow forced by a large-scale mean gradient, potential vorticity anomalies depend strongly on the properties of the surface (or at least near-surface) buoyancy anomalies.

To assess the validity of (14), we examine the results of a simulation of a nonlinear baroclinic unstable flow in a zonal $\beta$-plane channel using a primitive equation (PE) model (see Rivière et al. 2004). The parameter settings correspond to the Antarctic Circumpolar Current (ACC), one of the regions of the real ocean where the idealized geometry and forcings that we use applies well (Karsten et al. 2002). Characteristics are the same

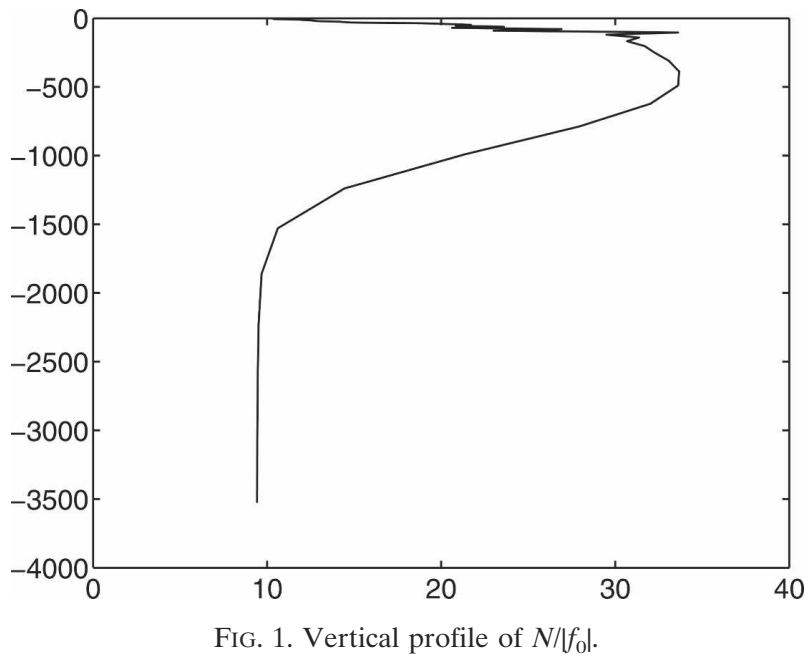

as in Rivière et al. (2004) except that the numerical resolution is $6 \mathrm{~km} \times 6 \mathrm{~km}$ in the horizontal and involves 35 levels in the vertical with a vertical grid spacing ranging from $5 \mathrm{~m}$ near the surface to $250 \mathrm{~m}$ near the bottom. We examine the dynamical fields after 50 days of simulation. The results, described throughout the paper, are qualitatively the same at a later or earlier time. The vertical profile of the mean Brunt-Väisälä frequency shows that the main thermocline is located at a depth around $500 \mathrm{~m}$ (Fig. 1). The mean Rossby number is Ro $=\sqrt{\left\langle\zeta^{2}\right\rangle} /\left|f_{0}\right| \approx 0.064$, where angle brackets are the horizontal averaging operator and $\zeta$ is the eddy relative vorticity. Relative vorticity reaches values as strong as $0.37\left|f_{0}\right|$. The Burger number is $\mathrm{Bu}=N^{2} H^{2} / f_{0}^{2} L^{2}$. Using $W / H=\sqrt{\left\langle(\boldsymbol{\nabla} \cdot \mathbf{u})^{2}\right\rangle}$ and, using the omega equation and thermal wind balance, it is easy to show that $W / H=$ $V^{2} f_{0} / H N^{2}=\left|f_{0}\right| \mathrm{Ro}^{2} \mathrm{Bu}^{-1}$ so that $\mathrm{Bu}=\mathrm{Ro}^{2}\left|f_{0}\right| /$ $\sqrt{\left\langle(\boldsymbol{\nabla} \cdot \mathbf{u})^{2}\right\rangle} \approx 2.44$. The simulation is thus close to the quasigeostrophic assumptions that we used in our analytical development. Figure 2a shows classical patterns associated with the evolution of a large-scale jet and with the presence of frontogenesis. Eddies of $150-\mathrm{km}$ diameter have developed on each side of the jet and have stirred filaments with high vorticities.

The strong resemblance between the PV field at 220 $\mathrm{m}$ and the surface buoyancy field (cf. Figs. $2 \mathrm{a}$ and $2 \mathrm{~b}$ ) gives a first indication of the relevance of relation (14). To quantify this further, we can examine the correlation in space between horizontal fields of eddy PV and its prediction (14). In view of (12a), $\alpha$ is computed from the regression of the horizontal field $f_{0} \partial_{y} \bar{Q}$ with that of $\partial_{y} \bar{b}_{s}$ at different levels, that is,

$$
\alpha(z)=\frac{\left\langle f_{0} \partial_{y} \bar{Q}_{y} \bar{b}_{s}\right\rangle}{\left\langle\left(\partial_{y} \bar{b}_{s}\right)^{2}\right\rangle} .
$$



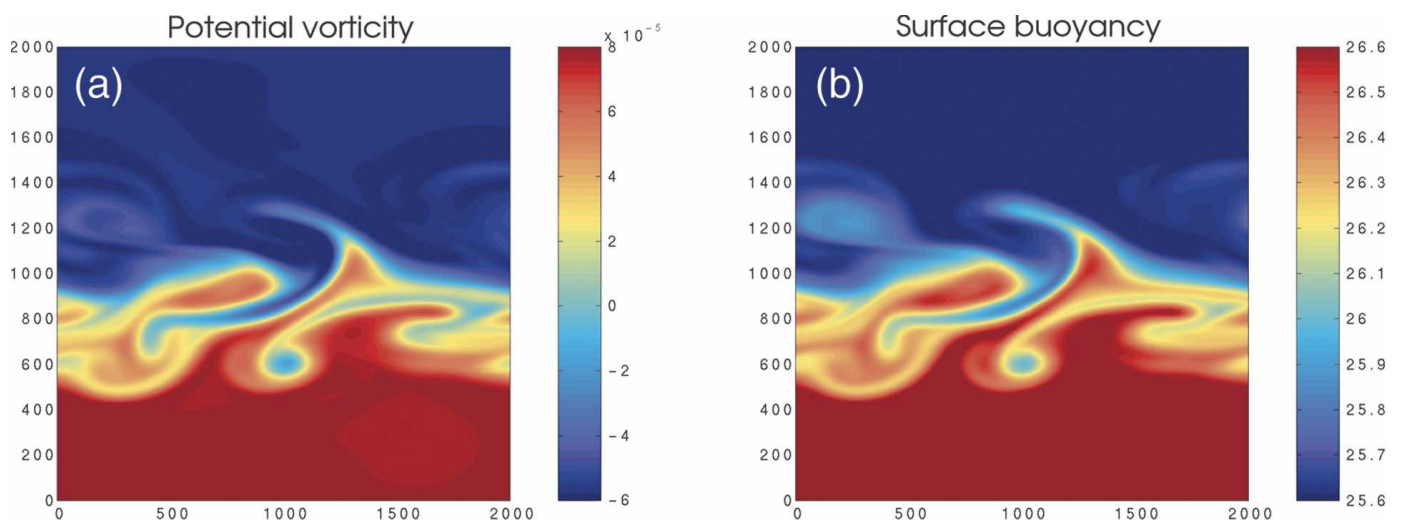

FIG. 2. (a) Potential vorticity field $\left(\mathrm{s}^{-1}\right)$ at $220-\mathrm{m}$ depth. (b) Surface buoyancy field (anomaly from mean $\rho_{0}=$ $\left.1000 \mathrm{~kg} \mathrm{~m}^{-3}\right)$. Horizontal scales are in kilometers.

The prediction of the eddy PV using (15) (see Fig. 3a) works remarkably well for the whole water column with a correlation close to 1 at $500 \mathrm{~m}$. It decreases where $\alpha$ vanishes, that is, around 700 and $1700 \mathrm{~m}$. The sign of $\alpha$ determines the sign of the correlation between the eddy PV and the surface buoyancy (note that $f_{0}<0$ in our parameter settings of the ACC). Such a correlation is negative in the upper layers and positive below. In our simulation, although the vertical scale of $N^{2}$ (Fig. 1) is larger than that of $\bar{b}$ below $100 \mathrm{~m}$, it is smaller in the first hundred meters. So one may be tempted to approximate $\alpha$ with only the vertical profile of $N^{2}$ [taking $F(z)=1$ ], that is,

$$
\alpha(z) \approx \frac{\partial}{\partial z}\left(\frac{f_{0}^{2}}{N^{2}}\right)
$$

Using (16) for $\alpha$ limits the validity of the prediction to the first three hundred meters and between 800 and $1700 \mathrm{~m}$ (see Fig. 3a).

The validity of (14) can also be evaluated by comparing $\alpha(z)$ given by (15) or (16) with the value obtained by regressing the eddy PV anomaly with surface buoyancy, that is,

$$
\frac{\left\langle f_{0} Q^{\prime} b_{s}^{\prime}\right\rangle}{\left\langle b_{s}^{\prime 2}\right\rangle}
$$

Each regression is actually an independent estimation of $\alpha(z)$ and should be close to each other if (14) is valid. This test allows one to determine the accuracy of the prediction of the amplitude of the PV anomalies. As expected, (15) and (17) agree with each other at all depths (Fig. 3b). However (16) gives, on the contrary, a
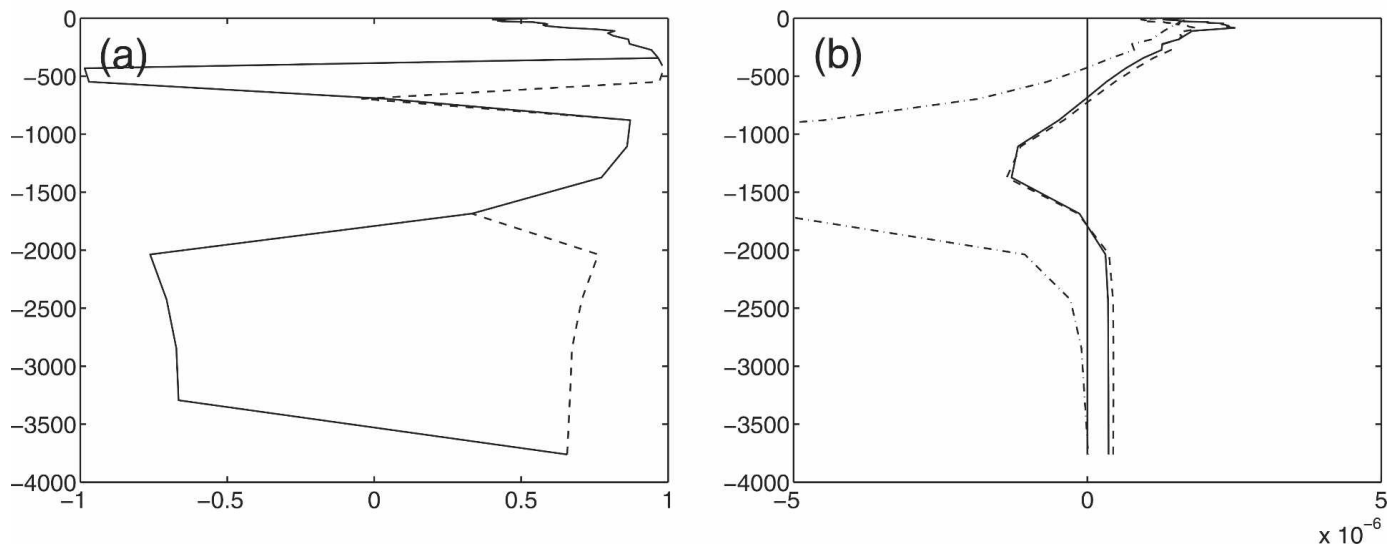

FIG. 3. (a) Spatial correlation as a function of depth of eddy PV and prediction by (14) using $\alpha$ from (15) (dashed curve) and using $\alpha$ from (16) (continuous curve). (b) Continuous curve: $\alpha$ computed from regression of PV with surface buoyancy, i.e., (17) as a function of depth; dashed curve: $\alpha$ computed from regression of large-scale gradients of PV and surface buoyancy, i.e., (15); dashed-dotted curve: $\partial\left(f_{0}^{2} / N^{2}\right) / \partial z$, i.e., (16). 
crude approximation: it disagrees with the other two below $100 \mathrm{~m}$ because it overestimates the amplitude of the PV field (Fig. 3b). Therefore the vertical structure of the buoyancy forcing needs to be taken into account to determine the right amplitude of the PV anomalies.

The initial setup of our experiment satisfies (11). However during the course of the simulation, one can expect that interaction between the large scales and the mesoscales will modify strongly relation (11) so that it may not hold exactly at a later time. Nevertheless, the different comparisons shown above indicate that relation (14) holds in our PE simulation of the nonlinear evolution of a baroclinic unstable jet. This demonstrates that PV anomalies are correlated with surface buoyancy anomalies and the explanation relies on the similar forcings by a mean gradient of the surface buoyancy and interior PV and on the action of the eddy field that stirs these tracers in the same manner.

\section{b. Cancellation of flows induced by the surface and by the interior}

The strong relation between eddy surface buoyancy and eddy interior PV has an impact on the decomposition into surface and interior solutions. This can be understood if we use the mathematical device introduced by Bretherton (1966), which shows that the surface buoyancy plays an equivalent role to the interior PV but with opposite sign. This opposite sign is responsible for the cancellation between the surface and interior solutions when adding the two solutions together.

From Bretherton's argument, one can formally introduce a thin layer (of height $\delta h>0$ ) on top of the ocean surface. At the top of this surface, one forces the buoyancy to vanish; that is, $b(z=\delta h)=0$. Then the associated potential vorticity is just

$$
\begin{aligned}
Q_{\text {bndry }} & =\left(\frac{1}{f_{0}}\right) \frac{\partial}{\partial z}\left(\frac{f_{0}^{2}}{N^{2}} b\right) \\
& =\left(\frac{1}{f_{0}} \frac{f_{0}^{2}}{N_{0}^{2}}\right) \frac{b(z=\delta h)-b(z=0)}{\delta h} \\
& =-\left(\frac{1}{f_{0}} \frac{f_{0}^{2}}{N_{0}^{2}}\right) \frac{b_{s}}{\delta h}
\end{aligned}
$$

since $b(z=0)=b_{s}$ by definition and $N_{0}=N(z=0)$. Taking the limit $\delta h \rightarrow 0$, the PV analog to the surface buoyancy is

$$
Q_{\text {bndry }}=-\left(\frac{1}{f_{0}} \frac{f_{0}^{2}}{N_{0}^{2}}\right) b_{s} \delta(z),
$$

where $\delta(z)$ is the Dirac function. This shows that if a positive correlation exists between PV in the upper layers and surface buoyancy, the PV sheet at the surface (induced by the surface buoyancy) will be in opposite phase with the nearby interior PV [cf. (14) with (19) for positive $\alpha$ ]. Therefore, when inverting PV, the interior solution $\psi_{\text {int }}$ and the surface solution $\psi_{\text {sur }}$ will tend to cancel each other because of this opposition.

To confirm this argument, we can examine the decomposition into surface and interior solution assuming that (14) holds. We can then rewrite solutions of (6a)(6b) and (7a)-(7b), defining $\varphi$ by

$$
\hat{\psi}=\frac{1}{f_{0}} \varphi \hat{b}_{s},
$$

where $\hat{\psi}$ and $\hat{b}_{s}$ are the two-dimensional (2D) Fourier transforms of $\psi$ and $b_{s}$. When decomposing $\psi$ into interior and surface solutions, we introduce $\varphi_{\text {sur }}$ and $\varphi_{\text {int }}$ for the surface and interior solutions, which satisfy

$$
-k^{2} \varphi_{\mathrm{int}}+\frac{\partial}{\partial z}\left(\frac{f_{0}^{2}}{N^{2}} \frac{\partial \varphi_{\mathrm{int}}}{\partial z}\right)=\alpha(z)
$$

with

$$
\left.\frac{\partial \varphi_{\text {int }}}{\partial z}\right|_{z=0}=0
$$

and

$$
-k^{2} \varphi_{\text {sur }}+\frac{\partial}{\partial z}\left(\frac{f_{0}^{2}}{N^{2}} \frac{\partial \varphi_{\text {sur }}}{\partial z}\right)=0,
$$

with

$$
\left.\frac{\partial \varphi_{\text {sur }}}{\partial z}\right|_{z=0}=1 .
$$

These variables depend only on $z$ and the spectral wavenumber $k=\left(k_{x}^{2}+k_{y}^{2}\right)^{1 / 2}$. Therefore, by $(20), \hat{\psi}_{\text {sur }}$ and $\hat{\psi}_{\text {int }}$ have the same phase given by that of $\hat{b}_{s}$, but the distribution on the vertical of their amplitude is given by $\varphi_{\text {sur }}$ and $\varphi_{\text {int }}$.

To exhibit these solutions, we choose to compute $\alpha(z)$ from (15). The numerical technique to solve (21a) through (22b) is standard (see Dritschel and Saravanan 1994, and references therein) and consists of adding a finite layer of PV equal to $b / \Delta z$ at the top. Figure $4 \mathrm{a}$ shows the contributions of the surface and the interior solutions to $\varphi_{\text {tot }}=\varphi_{\text {sur }}+\varphi_{\text {int }}$. Near the surface, that is, in the first $500 \mathrm{~m}, \varphi_{\text {sur }}$ and $\varphi_{\text {int }}$ have opposite signs and tend to cancel each other. This confirms the fact that the equivalence of the surface buoyancy to a Dirac function in PV with opposite sign gives rise to an anticorrelation between the surface and the interior solutions $\psi_{\text {sur }}$ and $\psi_{\text {int }}$ after the inversion. Also $\varphi_{\text {int }}$ represents $30 \%$ of $\varphi_{\text {sur }}$ and cannot be neglected in the upper layers. Because of the opposite sign between the interior and the surface solutions and the nonsmallness of 

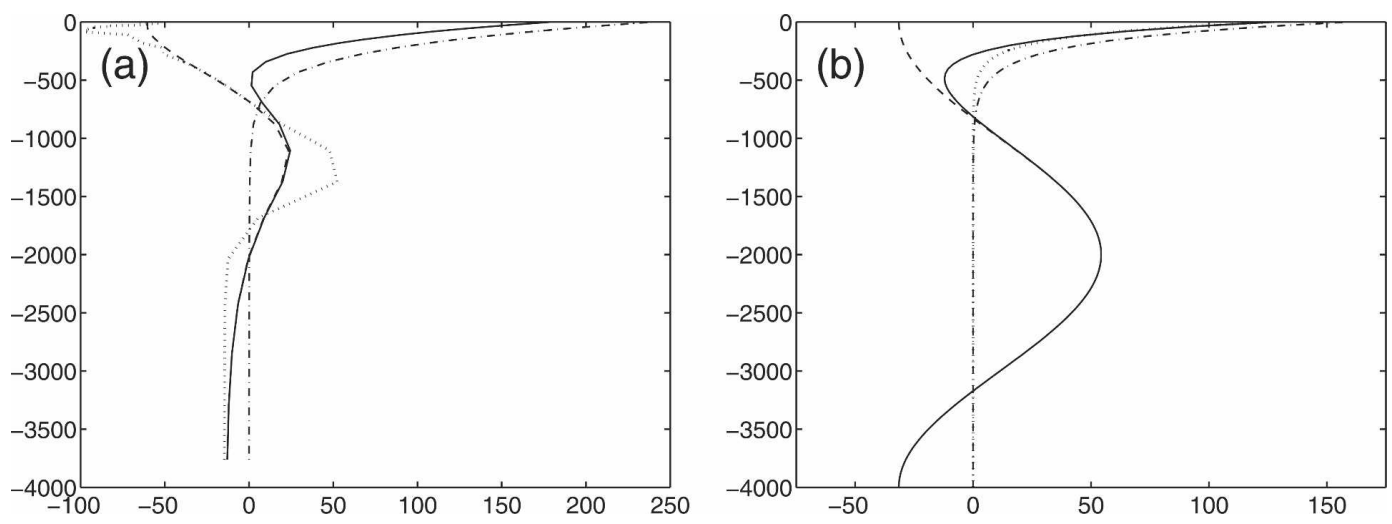

FIG. 4. (a) Vertical profiles of $\varphi$ (continuous curve), $\varphi_{\text {int }}$ (dashed curve), and $\varphi_{\text {sur }}$ (dashed-dotted curve) as a function of depth for a wavelength of $30 \mathrm{~km}$. The dotted curve represents $-\alpha(z) / k^{2}$. (b) Same quantities for the analytical expression (27). The dotted curve represents an exponential decaying function with $N /\left|f_{0}\right|=42$.

the interior solution near the surface, the total solution decreases much faster than the surface solution. Below $1000 \mathrm{~m}$ it is the interior solution that dominates; that is, the surface PV sheet does not affect the dynamics underneath the thermocline.

The relative magnitude of the surface and the interior solutions in the upper layers can be explained by comparing the interior PV with that from the surface buoyancy [using (19)]. The total PV in the upper layers can be decomposed as (e.g., Juckes 1994)

$$
\int_{z_{0}}^{0} Q_{\text {tot }}^{\prime} d z=\int_{z_{0}}^{0} Q_{\text {int }}^{\prime} d z+\int_{z_{0}}^{0} Q_{\text {bndry }} d z
$$

with $z_{0}<0$. The first term represents the contribution of the interior PV and the second term represents the contribution of the surface buoyancy. If we vertically integrate the first term using (14) and (12b), we have

$$
\begin{aligned}
\int_{z_{0}}^{0} Q_{\text {int }}^{\prime} d z & \approx \frac{1}{f_{0}} b_{s} \int_{z_{0}}^{0} \alpha d z \\
& \approx f_{0} N_{z=0}^{-2}\left(1-F_{z=z_{0}} \frac{N_{z=0}^{2}}{N_{z=z_{0}}^{2}}\right) b_{s},
\end{aligned}
$$

whereas the second term gives

$$
\int_{z_{0}}^{0} Q_{\text {bndry }} d z=-\frac{1}{f_{0}} \int_{z_{0}}^{0} \frac{f_{0}^{2}}{N^{2}} b_{s} \delta(z) d z=-f_{0} N_{z=0}^{-2} b_{s} .
$$

The integral in the first term uses the definition (12b) with $F(z=0)=1$. The term $1-F_{z=z_{0}} N_{z=0}^{2} / N_{z=z_{0}}^{2}$ is positive and smaller than 1 since $N^{2}(z)>N_{z=0}^{2}$ and $0<$ $F(z)<1$ in this region. This has two consequences: first, the surface solution will dominate down to the main thermocline (i.e., in the upper $500 \mathrm{~m}$ ). However, the interior solution may still be important (as observed) because of the smallness of $F_{z=z_{0}} N_{z=0}^{2} / N_{z=z_{0}}^{2}$, so that the surface and the interior contributions to the PV integral may have comparable amplitude.

We thus see that there are two ingredients that promote the strong relation between the surface and interior solutions. The first one is linked to the structure of the large-scale baroclinically unstable flow: the strong correspondence between the large-scale surface buoyancy and PV in the interior leads to a strong correlation between the eddy surface buoyancy and eddy PV within the upper layers. The second one, based on Bretherton's (1966) argument, is the dynamical equivalence of the eddy surface buoyancy to a PV sheet with sign opposite to that of the eddy PV in the interior. The two ingredients lead to the anticorrelation between the interior and surface solutions in the upper layers. Another remarkable consequence [as shown by (20), (21a)-(21b), and (22a)-(22b)] is that both solutions, and therefore the total solution, can be determined only from the surface buoyancy and the basin-scale characteristics of the vertical stratification captured by $N^{2}(z)$ and $\alpha(z)$.

\section{Application: Reconstructing 3D eddy dynamics}

Besides their strong anticorrelation, the surface and interior solutions display a rapid decay with depth, whose characteristics are set up by $N^{2}(z)$ and $\alpha(z)$. These properties and the dominance of the surface solution (whose structure is that of a SQG solution) strongly suggest the possibility to retrieve the 3D dynamics in these layers from the knowledge of the surface buoyancy alone and through a model similar to the SQG model (Blumen 1978; Hakim et al. 2002; Held et al. 1995), which is much simpler than the system (21a)(21b) and (22a)-(22b). Let us first discuss the vertical 
structure of the total solution through an analytical example.

\section{a. An analytical example}

The sharp decrease with depth of the total solution in the first $500 \mathrm{~m}$, revealed by Fig. 4a, suggests that it can be represented by a single exponential. This can be understood from an analytical example that assumes $N /\left|f_{0}\right|$ constant in these upper layers and that $\alpha(z)$ projects only on the barotropic and first baroclinic modes,

$$
\alpha(z) \approx A+B \cos \frac{2 \pi z}{H},
$$

where $A$ and $B$ are appropriate constants [with $A=-5$ $\times 10^{-7} \mathrm{~m}^{-1}, B=2 \times 10^{-6} \mathrm{~m}^{-1}$ using the profile given by (15)] and $H=4000 \mathrm{~m}$ the depth of the domain. In that case the total solution of the system (21a)-(21b) and (22a)-(22b) simply writes

$$
\begin{aligned}
\varphi(k, z) \approx & -\frac{A}{k^{2}}-\frac{B}{k^{2}+R_{d}^{-2}} \cos \left(\frac{2 \pi z}{H}\right) \\
& +\frac{\left|f_{0}\right|}{k N} \exp \left(\frac{k N z}{\left|f_{0}\right|}\right),
\end{aligned}
$$

with $R_{d}$ being the deformation radius $\left(R_{d}=20 \mathrm{~km}\right)$. The first two terms represent the interior solution and the last one the surface solution. Comparison of Figs. 4a and $4 \mathrm{~b}$ shows that this analytical expression displays in the upper layers the same characteristics as those obtained using the real $N^{2}(z)$ and $\alpha(z)$. Then one may be tempted to parameterize the $z$ dependence of the total solution as a single exponential, $\exp \left(k N_{0} z /\left|f_{0}\right|\right)$, with $N_{0}$ having a constant value (i.e., independent of $k$ and $z$ ) because of its fast decay scale. To find the correct $N_{0}$, we use (27) and compute the vertical derivative of $\left(\left|f_{0}\right| /\right.$ $k) \log \varphi(k, z)$ at $z=0$. We then average the result over a range of length scales between 30 and $150 \mathrm{~km}$; that is,

$$
\begin{aligned}
N_{0} & =\left\langle\left.\frac{\left|f_{0}\right|}{k} \frac{\partial \log \varphi(k, z)}{\partial z}\right|_{z=0}\right\rangle \\
& =\left\langle\frac{N}{1-\frac{k N}{\left|f_{0}\right|}\left(\frac{A}{k^{2}}+\frac{B}{k^{2}+R_{d}^{-2}}\right)}\right\rangle
\end{aligned}
$$

with angle brackets denoting the averaging operator over $k$ between $2 \pi /\left(150 \times 10^{3}\right) \mathrm{m}^{-1}$ and $2 \pi /\left(30 \times 10^{3}\right)$ $\mathrm{m}^{-1}$. This gives a value $N_{0} /\left|f_{0}\right|=42$, which scales well with the total solution in the first $300 \mathrm{~m}$ (Fig. 4b). This analytical development illustrates that the total solution decreases much more rapidly than the surface solution alone (for which $N /\left|f_{0}\right|=30$ in this example) because of the partial cancellation between the interior and surface solution.
The use of an approximated solution (involving an exponential) instead of the total solution (i.e., $\phi_{\text {tot }}$ ) is motivated by the very rapid decay of the solution. It is also based on the fact that different studies (Juckes 1994; Wirth et al. 1997) have shown that a model with constant $N_{0}$ is able to reproduce the dynamical features with some realism.

\section{b. An "effective" surface $Q G$ solution}

One could in principle solve (21a)-(21b) and (22a)(22b) numerically and be able to reconstruct the 3D velocity field using (20) and an omega equation (Hoskins et al. 1978). However such a method is numerically expensive since it involves inverting a three-dimensional elliptic operator to retrieve the vertical velocity field. Instead we propose to approximate further the problem, motivated by the good approximation obtained for the analytical example. This leads us to reduce the full problem (21a)-(21b) and (22a)-(22b) to an "effective" SQG model.

To show that the analytical example provides a good template for the full problem, we can try to approximate $\varphi_{\text {int }}$. Assuming that $\alpha(z)$ mostly projects on the first vertical modes, one can find a specific solution of (21a)-(21b) and (22a)-(22b) for submesoscale motions (for which $k^{2} \gg R_{d}^{-2}$ ). Then the interior solution $\varphi_{\text {int }}$, using the same derivation as above, can be approximated by

$$
\varphi_{\text {int }}(k, z)=-\frac{\alpha(z)}{k^{2}} .
$$

An inspection of Fig. 4a shows that the shape is correct down to $700 \mathrm{~m}$ for a length scale of $30 \mathrm{~km}$. This shows that we can use the analytical example of section 4 a to derive our effective SQG model. We thus suggest that a reduced model with an exponential decay can grasp the essential property of (21a)-(21b) and (22a)-(22b). In other words, an effective SQG model, using a constant $N_{0}$ to take into account both the interior and surface solutions, should be able to reconstruct the $3 \mathrm{D}$ balanced motions of the PE simulation in the upper oceanic layers from just the knowledge of the surface buoyancy. Such a model with constant $N_{0}$ has been thoroughly investigated in the past (Blumen 1978; Hakim et al. 2002; Held et al. 1995; Juckes 1994) and this is one of the reasons we propose to use it.

To evaluate the value of $N_{0}$ (independent of $k$ and $z$ ), we have chosen the following general procedure that requires only the knowledge of $N^{2}(z)$ and $\alpha(z)$ : we compute solutions $\varphi_{\text {tot }}=\varphi_{\text {int }}+\varphi_{\text {sur }}$ given $N^{2}(z), \alpha(z)$ from (15) and using relations (21a)-(21b) and (22a)-(22b). We only consider length scales between 30 and $150 \mathrm{~km}$, 
which correspond to a range of submeso- to mesoscales for which the SQG theory should be relevant. Then we do a linear regression between $\left|f_{0}\right| \log \left[\varphi_{\text {tot }}(k, z) / \varphi_{\text {tot }}(k\right.$, $\left.\left.z_{0}\right)\right] / k$ and $z$ for $z$ varying between $z=-110 \mathrm{~m}$ and $z=$ $-343 \mathrm{~m}$, corresponding to a range where the exponential fit should work. The reason for not using depths between the surface and $110 \mathrm{~m}$ is that we prefer not to use data in the mixed layer. The final step is to average the regression coefficient for the different length scales considered. This gives a value, $N_{0} /\left|f_{0}\right| \approx 45$. We follow a similar approach to find the amplitude of the exponential solution: we want to approximate $\varphi_{\text {tot }}$ by

$$
\varphi_{\text {tot }}(k, z) \approx c \frac{\left|f_{0}\right|}{N_{0} k} \partial_{z} \varphi_{\text {tot }}\left(k, z_{0}\right) \exp \left[\frac{N_{0} k}{\left|f_{0}\right|}\left(z-z_{0}\right)\right]
$$

with $c$ a constant (independent of $k$ and $z$ ). To determine $c$ we do a regression between $\left(\left|f_{0}\right| / N_{0} k\right) \partial_{z} \varphi_{\text {tot }}(k$, $\left.z_{0}\right) \exp \left[\left(N_{0} k /\left|f_{0}\right|\right)\left(z-z_{0}\right)\right]$ and $\varphi_{\text {tot }}(k, z)$ for length scales between 30 and $150 \mathrm{~km}$ and depths between 110 and $343 \mathrm{~m}$. We find $c \approx 1.12$. Again, this general procedure to find the constant values of $N_{0}$ and $c$ requires only the knowledge of $N(z)$ and $\alpha(z)$.

As shown in Fig. 5, the fit using $N_{0} /\left|f_{0}\right|=45$ and $c \approx$ 1.12 works rather well in the first few hundred meters for a range of length scales between 50 and $100 \mathrm{~km}$. Below (above) this range, the amplitude is underestimated (overestimated) but the decay scale is correct (not shown). While neither the surface solution nor the interior solution alone, computed from the real $N^{2}(z)$ and $\alpha(z)$, could simulate the total solution $\varphi_{\text {tot }}$, an effective surface QG solution with a proper constant $N_{0}$ (i.e., independent of $k$ and $z$ ) is thus able to predict the correct shape of the solution $\varphi(k, z)$ in the first $500 \mathrm{~m}$.

We can briefly review the general properties of the resulting effective SQG model with constant $N_{0}$ [for a developed study, see Held et al. (1995) and Hakim et al. (2002)]. In the case of a semi-infinite vertical plane, solutions of (7a)-(7b) using (31) and (20) can be found using spectral horizontal transforms. For each wavenumber $k$, the solution is

$$
\begin{aligned}
& \hat{b}_{s}=\hat{b}\left(k, z_{0}\right) \exp \left(-\frac{N_{0} k z_{0}}{\left|f_{0}\right|}\right), \\
& \hat{b}=\hat{b}_{s} \exp \left(\frac{N_{0} k z}{\left|f_{0}\right|}\right), \text { and } \\
& \hat{\psi}=\operatorname{sign}\left(f_{0}\right) \frac{c}{N_{0}} \frac{\hat{b}_{s}}{k} \exp \left(\frac{N_{0} k z}{\left|f_{0}\right|}\right),
\end{aligned}
$$

where the caret again denotes the Fourier transform and $\operatorname{sign}()$ is the sign function. Equation (31) was used

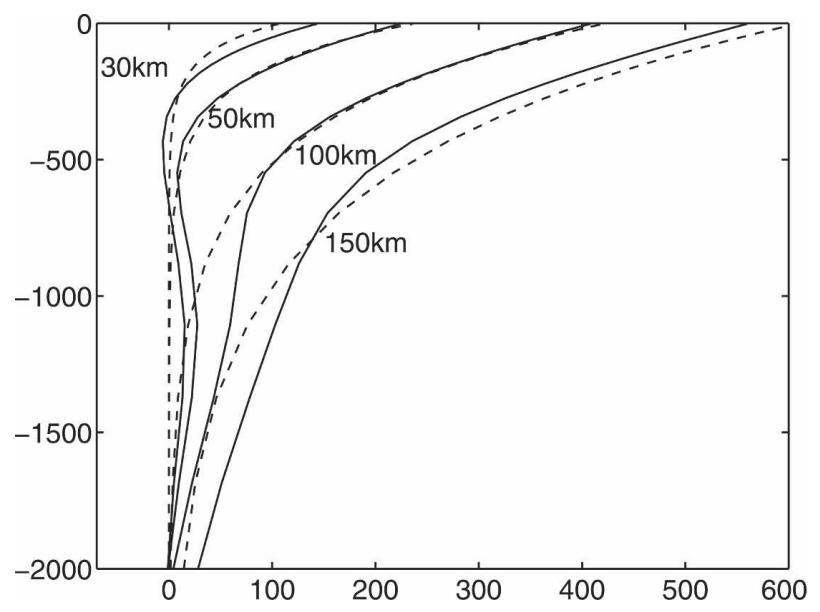

FIG. 5. Vertical profiles of $\varphi$ (continuous curves) and of its exponential fit using $N /\left|f_{0}\right|=45$ (dashed curves) for different wavelengths $(30,50,100$, and $150 \mathrm{~km})$.

to derive (32c). From the buoyancy equation, one can deduce the vertical velocity $w$ at any depth, using spectral transforms:

$$
\begin{aligned}
\hat{w} & =-\frac{c^{2}}{N_{0}^{2}} \frac{\widehat{D b}}{D t} \\
& =-\frac{c^{2}}{N_{0}^{2}}\left\{\partial_{t}\left[\hat{b}_{s} \exp \left(\frac{N_{0} k z}{\left|f_{0}\right|}\right)\right]+\widehat{J(\psi, b)}\right\}, \quad \text { and } \\
& =-\frac{c^{2}}{N_{0}^{2}}\left[-\widehat{J\left(\psi_{s}, b_{s}\right.} \exp \left(\frac{N_{0} k z}{\left|f_{0}\right|}\right)+\widehat{J(\psi, b)}\right]
\end{aligned}
$$

[The last step uses the fact that $\partial_{t} b_{s}+J\left(\psi_{s}, b_{s}\right)=0$.] Given that the amplitude of the streamfunction is proportional to $c / N_{0}$, this leads to a dependence on $c^{2}$ for the amplitude of the vertical velocity. This could be consistent with the findings of Viudez and Dritschel (2004) and others who find that the QG vertical velocity is, in general, in phase with the PE vertical velocity but with smaller amplitude. In this framework, all dynamical quantities can be deduced from the surface buoyancy field. The reason is that the zero PV constraint (7a) forces vertical scales to be dependent on horizontal ones.

\section{c. Comparison of the effective $S Q G$ solution with the PE simulation}

We can assess the pertinence of the effective SQG model through the examination of different dynamical quantities of the PE simulation and of their prediction with the effective SQG model. We have made the domain doubly periodic by symmetry along the north edge of the channel. We choose to take a reference level at $z=z_{0}=-220 \mathrm{~m}$, underneath the mixed layer present in our PE simulation. The reason stems from 

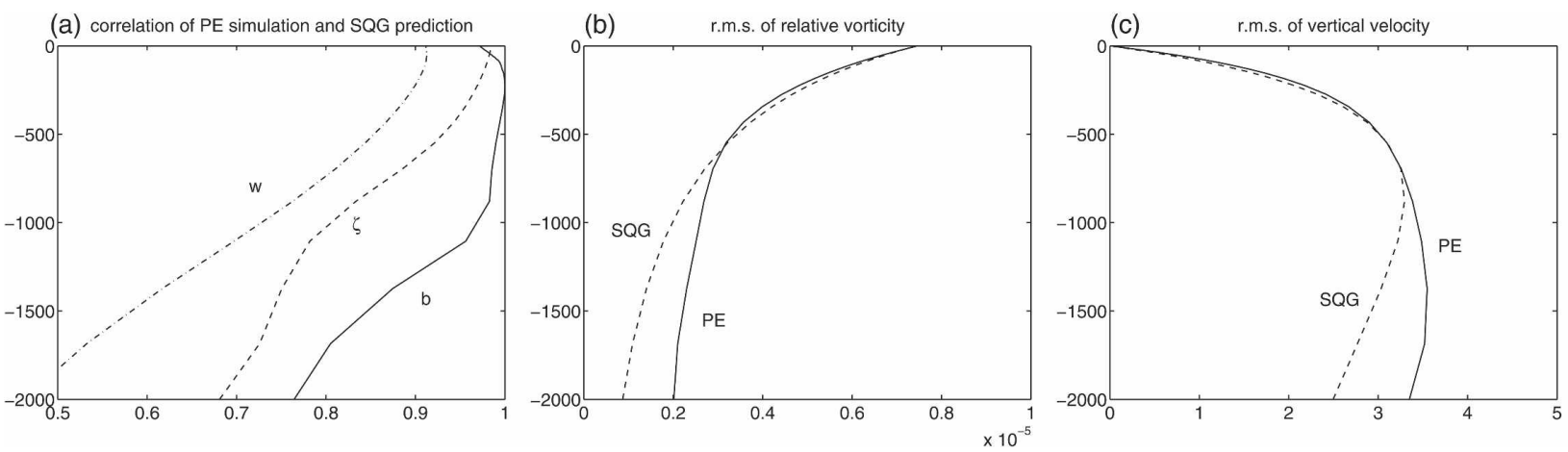

FIG. 6. (a) Spatial correlations between observed quantities of the PE simulation and prediction by the effective SQG model, as a function of depth. Continuous curve: eddy density (anomaly relative to the zonal mean); dashed curve: eddy vorticity; dashed-dotted curve: eddy vertical velocity. (b) Rms of eddy vorticity $\left(\mathrm{s}^{-1}\right)$ as a function of depth. Continuous curve: PE simulation; dashed curve: effective SQG prediction. (c) Rms of eddy vertical velocity $\left(\mathrm{m} \mathrm{day}^{-1}\right)$ as a function of depth [curves with same definition as (b)].

the fact that, in the mixed layer, buoyancy is homogenized vertically so that quasigeostrophic PV is infinite $(N \approx 0)$. From the total surface buoyancy field (zonal mean plus anomaly), we compute at different vertical levels the predicted eddy buoyancy from (32b), relative vertical vorticity $\zeta=\nabla^{2} \psi$ from (32c), and vertical velocity from (33).

The spatial correlations between the different dynamical quantities from the PE simulation and their prediction by the effective SQG model are quite good, as shown in Fig. 6a. The prediction of the eddy density in the interior is nearly perfect in the first $1000 \mathrm{~m}$ as the correlation is larger than 0.95 .

In the first $500 \mathrm{~m}$, the vorticity correlation is larger than 0.9 , which is also excellent. A more difficult quantity to predict is the vertical velocity, as it is a nonlinear function of buoyancy by (33). We obtain a spatial correlation greater than 0.85 in the first $500 \mathrm{~m}$, which demonstrates that the effective SQG model is able to reconstruct a 3D dynamical field consistent with the full primitive equation simulation. Other diagnostics confirm the pertinence of this model, such as the horizontal root mean square of vorticity and vertical velocity as a function of depth (Figs. 6b,c). In the first $500 \mathrm{~m}$, the effective SQG model predicts the correct magnitude. Below, the magnitude decreases too rapidly because the model does not take into account the energy of the interior flow. Its region of validity thus seems to be the upper $500 \mathrm{~m}$.

As final comparisons, one can examine the spatial distributions of vorticity and vertical velocity and their respective predictions (Fig. 7). From just the knowledge of the surface buoyancy, the effective SQG model remarkably reproduces all the features present in the vorticity field (Fig. 7b). This confirms the relation between vorticity (or the streamfunction) and buoyancy as ex- pressed in (32c). Small differences can be observed at small scales. They can be interpreted in the light of Fig. 5 , which showed that, for a certain depth, the exponential fit fails first at small scales. The vertical velocity field (Fig. 7c) displays strong vertical motions on the edges of submesoscale fronts, which can reach up to 32 $\mathrm{m}$ day $^{-1}$ at $z=-430 \mathrm{~m}$. We observe dipoles associated with upward and downward motions on each side of filaments, corresponding to cross-frontal circulations. Such structures are well reproduced by the prediction of the vertical velocity field (Fig. 7d). Most of the submesoscale features are present and only the very small scales are not well predicted by the model.

This technique, using the effective SQG model, has a strong advantage relative to the traditional technique that solves the omega equation (Hoskins et al. 1978) or other more elaborated methods (Viudez and Dritschel 2004). The omega equation relates by a three-dimensional elliptic operator the vertical velocity to the horizontal buoyancy gradient and velocity gradient. In observational studies, one needs a rather good threedimensional sampling in order to represent accurately the strong buoyancy gradients at any depth. As compared with the traditional technique, our technique depends only on the sampling of the horizontal buoyancy field at the surface and is much simpler to use.

\section{Discussion}

We have shown that large-scale unstable baroclinic flows possess the property of having potential vorticity mesoscale anomalies at depth correlated with surface buoyancy-or density-anomalies. Indeed, it is the structure of the large-scale forcing, in particular the similarity between the large-scale PV and surface density forcings, that leads to this correlation. One impor- 
(a) PE relative vorticity at $430 \mathrm{~m}$

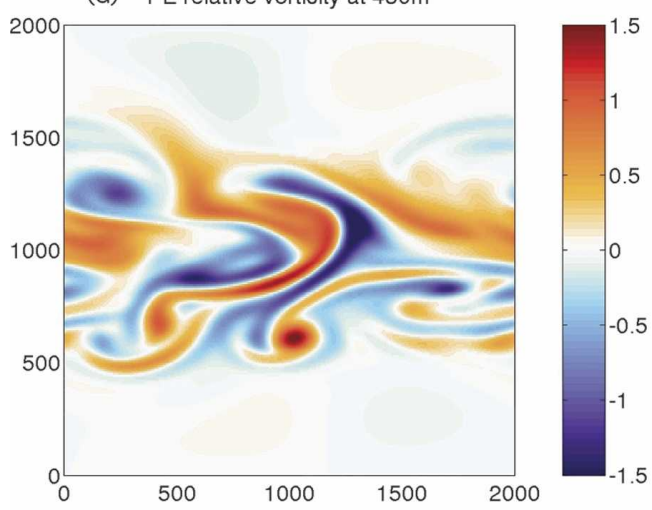

(c) PE vertical velocity at $430 \mathrm{~m}$

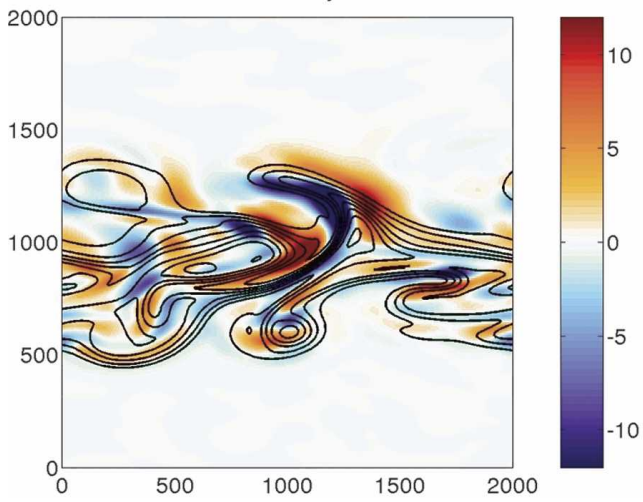

(b) SQG prediction of relative vorticity at $430 \mathrm{~m}$

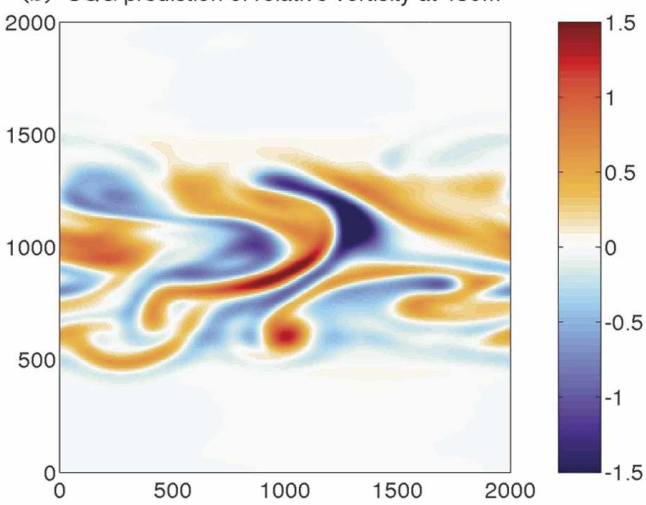

(d) SQG prediction of vertical velocity at $430 \mathrm{~m}$

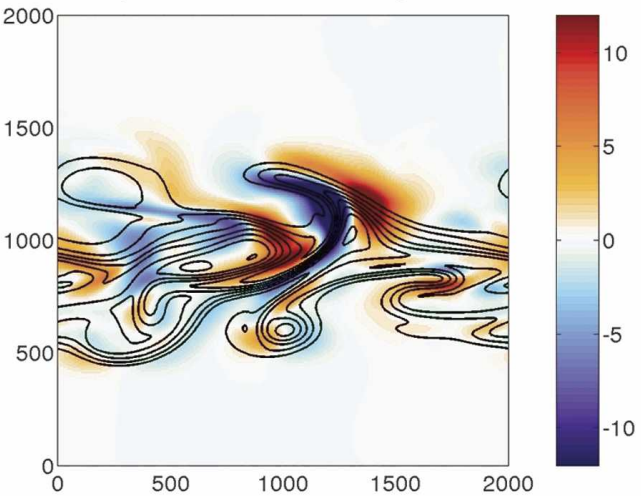

FIG. 7. (top) Vertical relative vorticity field at $430 \mathrm{~m}\left(10^{-5} \mathrm{~s}^{-1}\right)$ : (a) PE simulation and (b) SQG prediction. (bottom) Vertical velocity field at $430 \mathrm{~m}\left(\mathrm{~m} \mathrm{day}^{-1}\right)$ : (c) PE simulation and (d) effective SQG prediction. Superimposed are the surface density contours.

tant consequence, deduced from the PV invertibility principle, is the strong anticorrelation between the upper-layer velocity field associated with the interior PV field and that associated with the surface density field. The other important consequence is that this $3 \mathrm{D}$ velocity field can be determined from just the surface density and the basin-scale characteristics of the vertical stratification by solving a 3D elliptic equation.

From these results, the possibility has been raised of diagnosing the dynamics from only the surface density using a simpler method based on the SQG theory. This method has been tested using a PE simulation of a baroclinic unstable current after its fully nonlinear evolution. We have shown that this method allows one to diagnose quite efficiently the 3D dynamics of mesoscale and submesoscale motions in the oceanic upper layers (the first $500 \mathrm{~m}$ ) from the surface density field.

There are different limitations of the method to reconstruct the 3D circulation from the surface density. First it only works in the upper layers (typically the first $500 \mathrm{~m}$ ) and it cannot work in the lower layers (contrary to standard PV inversion techniques). It also depends quite strongly on the type of forcing that generates the flow. We expect it to work when the instability is driven by large-scale meridional gradients. Also the method will not diagnose three-dimensional circulation associated with dynamical structures that do not possess a signature at the ocean surface.

One application of this method would be to get the dynamical field of the upper layers (in particular the vertical velocity field) from satellite images, such as infrared images, insofar as temperature is the main surface density contribution. However the surface temperature results not only from the influence of mesoscale eddies but also from the dynamics of the surface mixed layer driven by atmospheric forcings. This method can work only when the mixed layer mesoscale and submesoscale structures strongly resemble those below the mixed layer. This should be the case for example just after a strong wind pulse. Further work is necessary to determine the situations where this method can be applied with satellite images. 
Acknowledgments. The authors thank Lien Hua, Pascal Rivière, and Anne-Marie Tréguier for helpful comments about this work. Also, Shafer Smith helped in improving substantially the manuscript. All simulations were performed on the supercomputers of CNRS at IDRIS. This work was supported by Institut Français de Recherche pour l'Exploitation de la Mer (IFREMER) and the Centre National de la Recherche Scientifique (CNRS).

\section{REFERENCES}

Bishop, C. H., and A. J. Thorpe, 1994: Potential vorticity and the electrostatics analogy: Quasigeostrophic theory. Quart. J. Roy. Meteor. Soc., 120, 713-731.

Blumen, W., 1978: Uniform potential vorticity flow. Part I: Theory of wave interactions and two-dimensional turbulence. J. Atmos. Sci., 35, 774-783.

Bretherton, F. P., 1966: Critical layer instability in baroclinic flows. Quart. J. Roy. Meteor. Soc., 92, 325-334.

Davis, C. A., 1993: Comments on "Decomposing the atmospheric flow using potential vorticity framework." J. Atmos. Sci., 50, 2065-2067.

_ , and K. A. Emanuel, 1991: Potential vorticity diagnostics of cyclogenesis. Mon. Wea. Rev., 119, 1929-1953.

Dritschel, D. G., and R. Saravanan, 1994: Three-dimensional quasigeostrophic contour dynamics, with an application to stratospheric vortex dynamics. Quart. J. Roy. Meteor. Soc., 120, 1267-1298.

Gent, P. R., and J. C. McWilliams, 1983: Consistent balanced models in bounded and periodic domains. Dyn. Atmos. Oceans, 7, 67-93.

Green, J. S. A., 1987: Comments on "On the use and significance of isentropic potential vorticity maps." Quart. J. Roy. Meteor. Soc., 113, 401-404.

Hakim, G. J., D. Keyser, and L. F. Bosart, 1996: The Ohio Valley wave-merger cyclogenesis event of 25-26 January 1978. Part II: Diagnosis using quasigeostrophic potential vorticity inversion. Mon. Wea. Rev., 124, 2176-2205.

, C. Snyder, and D. J. Muraki, 2002: A new surface model for cyclone-anticyclone asymmetry. J. Atmos. Sci., 59, $2405-$ 2420.
Held, I. M., R. T. Pierrehumbert, S. T. Garner, and K. L. Swanson, 1995: Surface quasi-geostrophic dynamics. J. Fluid Mech., 282, 1-20.

Holopainen, E., and J. Kaurola, 1991: Decomposing the atmospheric flow using potential vorticity framework. J. Atmos. Sci., 48, 2614-2625.

Hoskins, B. J., I. Draghici, and H. C. Davies, 1978: A new look at the $\omega$-equation. Quart. J. Roy. Meteor. Soc., 104, 31-38.

— M. E. McIntyre, and A. W. Robertson, 1985: On the use and significance of isentropic potential vorticity maps. Quart. J. Roy. Meteor. Soc., 111, 877-946.

Hua, B. L., and D. B. Haidvogel, 1986: Numerical simulations of the vertical structure of quasi-geostrophic turbulence. J. Atmos. Sci., 43, 2923-2936.

Juckes, M., 1994: Quasigeostrophic dynamics of the tropopause. J. Atmos. Sci., 51, 2756-2768.

Karsten, R., H. Jones, and J. Marshall, 2002: The role of eddy transfer in setting the stratification and transport of a circumpolar current. J. Phys. Oceanogr., 32, 39-54.

Klein, P., A. Tréguier, and B. L. Hua, 1998: Three-dimensional stirring of thermohaline fronts. J. Mar. Res., 56, 589-612.

McIntyre, M. E., and W. A. Norton, 2000: Potential-vorticity inversion on a hemisphere. J. Atmos. Sci., 57, 1214-1235.

Pedlosky, J., 1987: Geophysical Fluid Dynamics. Springer-Verlag, $710 \mathrm{pp}$.

Rivière, P., A.-M. Tréguier, and P. Klein, 2004: Effects of bottom friction on nonlinear equilibration of an oceanic baroclinic jet. J. Phys. Oceanogr., 34, 416-432.

Robinson, W. A., 1989: On the structure of potential vorticity in baroclinic instability. Tellus, 41A, 275-284.

Smith, K. S., and G. K. Vallis, 2001: The scales and equilibration of midocean eddies: Freely evolving flow. J. Phys. Oceanogr., 31, 554-571.

Vallis, G. K., 1996: Potential vorticity inversion and balanced equations of motion for rotating and stratified flows. Quart. J. Roy. Meteor. Soc., 122A, 291-322.

Viudez, A., and D. G. Dritschel, 2004: Dynamic potential vorticity initialization and the diagnosis of mesoscale motion. J. Phys. Oceanogr., 34, 2761-2773.

Wirth, V., C. Appenzeller, and M. Juckes, 1997: Signatures of induced vertical air motion accompanying quasi-horizontal roll-up of stratospheric intrusions. Mon. Wea. Rev., 125, 2504-2519. 